\title{
Review of: "Characterization and complete genome sequence of bacteriophage vB_Vc_SrVc2, a marine phage that infects Vibrio campbellii"
}

\author{
Arun Dhar ${ }^{1}$ \\ 1 University of Arizona
}

Potential competing interests: The author(s) declared that no potential competing interests exist.

The authors isolated and sequenced the genome of a lytic phage, vB_Vc_SrVc2 from white shrimp hepatopancreas displaying clinical signs of acute hepatopancreatic necrosis disease (AHPND). The viral genome is $\sim 43 \mathrm{~kb}$ with a GC content of $49.2 \%$ and encodes 49 putative ORFs with no tRNAs. Compared to a similar virus, SrVc9 previously sequenced by the same group of researchers, SrVc2 showed three single nucleotide polymorphisms, two small deletions and one nucleotide insertion and display slightly different infectivity profiles. The virus SrVc2 is resistant to broad range of temperatures, salinities, UV radiations and chloroform, and has a high lytic ability against Vibrio isolates. The authors described the new virus as a member of the genus Maculvirus, family Autographiviridae.

The authors need to address the following queries before the manuscript is considered for publication.

1. Abstracts: The authors describe the virus as a member of the genus Maculvirus, family Autographiviridae. The conclusion is based on genome sequence similarity to a very similar virus, SrVc9. The conclusion should indicate that the taxonomic assignment is a proposition and is not definitive until ICTV approves it. So the abstract needs be edited to reflect this proposition.

2. The authors have published a paper describing the genome sequence of a virus, SrVc9 which is very similar to the virus described here (J Appl Microbiol https://doi.org/10.1111/jam.14937). However, in the introduction there is hardly any description of their previous work. The introduction needs to describe their previous work and describe how the current work is adding new information to their previous work instead of avoiding a discussion of their previous work altogether. Otherwise, it is difficult to comprehend how they came to a definitive conclusion about the taxonomy of this virus.

3. It is interesting to note that the two viruses, SrVc2 and SrVc9 have very little differences yet there are some differences in terms of their biological activities. I would propose the authors summarize the differences in the biological properties of these two viruses in Table 1, and then discuss the differences in their activities in light of the genomic differences. This may help to correlate the genomic differences to biological differences.

4. Fig. 4. How do the authors explain a sudden drop in phage survival at $20 \mathrm{ppt}$ ? Did the author repeat the 
assay independently? If not, they are highly encouraged to repeat the assay just to make sure that sudden drop in phage survival at 20 ppt was not due to a technical error. In their previously published paper, sensitivity of the phages to salinity change at 20 ppt was not so dramatic (See Fig. 1C in https://doi.org/10.1111/jam.14937).

To summarize, the authors published the genome sequence of a virus which is very similar to a virus recently reported (https://doi.org/10.1111/jam.14937). Interestingly, instead of discussing the genomic and biological difference between the two viruses, they went ahead and discussed only one of them, SrVc2 (the current virus, and not the previously published virus SrV(9). The manuscript needs to be revised and discussions revised comparing the two viruses. I think that would make this paper more interesting. 